\title{
Thank You Reviewers!
}

\author{
Karin Milovanovic-Hanselman
}

Correspondence:

milovanovic@fsw.eur.nl Erasmus University Rotterdam, Rotterdam, The Netherlands

\section{Contributing reviewers}

The Board of Comparative Migration Studies would like to use this opportunity to thank our reviewers for their continued support. Their expertise and hard work as a reviewer is invaluable to us and has helped the authors in improving their manuscripts.

The reviewers' work is extremely important for us to publish high quality papers. We would like to recognize the reviewers' contributions by publishing their names.

$\begin{array}{lll}\text { Maria Abascal } & \text { Simon Green } & \text { Joana Sousa Ribeiro } \\ \text { Joaquin Arango } & \text { Anniken Hagelund } & \text { Christof Roos } \\ \text { Can Aybek } & \text { Yossi Harpaz } & \text { Dumitru Sandu } \\ \text { Martin Bak-Jorgensen } & \text { Claire Healy } & \text { Simone Scarpa } \\ \text { Michaela Benson } & \text { Jonas Hinnfors } & \text { Jens Schneider } \\ \text { Christophe Bertossi } & \text { Claus Hofhansel } & \text { Peter Scholten } \\ \text { Pieter Bevelander } & \text { Nils Holtug } & \text { Giuseppe Sciortino } \\ \text { Joachim Blatter } & \text { Marc Hooghe } & \text { Sam Scott } \\ \text { Caroline Bledsoe } & \text { Thomas Huddleston } & \text { Deniz Sert } \\ \text { Paolo Boccagni } & \text { Tomás Jiménez } & \text { Emily Skop } \\ \text { Elisa Brey } & \text { Christian Joppke } & \text { J. Slootjes } \\ \text { Kathy Burrell } & \text { Eleonore Kofman } & \text { Andrea Spehar } \\ \text { Jean Pierre Cassarino } & \text { Holger Kolb } & \text { Sarah Spencer } \\ \text { Héctor Cebolla } & \text { Arjen Leerkes } & \text { Andrei Stavila } \\ \text { Manlio Cinalli } & \text { John Lie } & \text { L.E. (Rens) Tacoma } \\ \text { Kathy Davis } & \text { Trine Lund Thomsen } & \text { Alexandre Tandé } \\ \text { Angeliki Dimitriadi } & \text { Anna Lundberg } & \text { Sorana Toma } \\ \text { Henrik Emilsson } & \text { Blanca Garces Mascarenas } & \text { Kate Torkington } \\ \text { Han Entzinger } & \text { Pablo Mateos } & \text { Anna Triandafyllidou } \\ \text { Michael Eve } & \text { Rahsaan Maxwell } & \text { Mark Van Ostaijen } \\ \text { Adrien Favell } & \text { Ines Michalowski } & \text { Floris Vermeulen } \\ \text { Allan Findlay } & \text { Liza Mugge } & \text { Sarah Wallace Goodman } \\ \text { Fenella Fleischmann } & \text { Pontus Odmalm } & \text { Catherine Wihtol de Wenden } \\ \text { A. Geddes } & \text { Ringo Ossewaarde } & \text { Ricard Zapata-Barrero } \\ \text { Robbie Gilligan } & \text { Dominic Pasura } & \text { Karin Zelano } \\ \text { Laura Gonzalez-Murphy } & \text { Irene Ponzo } & \text { Malisa Zobel } \\ \text { Elzbieta Gozdziak } & \text { Rogier Reekum } & \\ \text { Izabella Grabowska } & \text { Niamh Reilly } & \\ & & \end{array}$

(c) The Author(s). 2017 Open Access This article is distributed under the terms of the Creative Commons Attribution 4.0 International License (http://creativecommons.org/licenses/by/4.0/), which permits unrestricted use, distribution, and reproduction in any medium, provided you give appropriate credit to the original author(s) and the source, provide a link to the Creative Commons license, and indicate if changes were made. 